Pacific Journal of Mathematics

RESTRICTIONS OF CERTAIN FUNCTION SPACES TO CLOSED
SUBGROUPS OF LOCALLY COMPACT GROUPS 


\title{
RESTRICTIONS OF CERTAIN FUNCTION SPACES TO CLOSED SUBGROUPS OF LOCALLY COMPACT GROUPS
}

\author{
Michael Cowling and Paul Rodway
}

\begin{abstract}
Let $G$ be a locally compact group, and $E(G)$ either the space $C_{u}(G)$ of bounded left and right uniformly continuous functions on $G$, the space $W(G)$ of weakly almost periodic functions on $G$, or the Fourier-Stieltjes algebra $B(G)$ of $G$. Let $\left.E(G)\right|_{H}$ be the space of restrictions of $E(G)$-functions to the closed subgroup $H$ of $G$. A necessary and sufficient condition is given for an $E(H)$-function to belong to $\left.E(G)\right|_{H}$ when $H$ is a normal subgroup of $G$. It is also shown that $\left.E(G)\right|_{H}$ is all of $E(H)$ when $H$ is any closed subgroup of a [SIN]-group. The techniques employed here can be used to deal with other function spaces.
\end{abstract}

Let $C(G)$ be the space of bounded continuous complex-valued functions on $G$ with the uniform norm, \|\|$_{\infty}$, and $\beta(G)$ be the StoneCech compactification of $G$, i.e., the maximal ideal space of $C(G)$, to which $C(G)$-functions extend naturally, via the Gelfand transform. The left translation operator is denoted $\lambda$ :

$$
[\lambda(g) u]\left(g^{\prime}\right)=u\left(g^{-1} g^{\prime}\right) \quad g, g^{\prime} G, u \in C(G) .
$$

The reader will recall that $u$ in $C(G)$ is called weakly almost periodic if the set $\lambda(G) u$ of left translates of $u$ is relatively compact in the weak topology of $C(G)$. Equivalently, one may require that for any sequence $\left\{g_{j}\right\}$ of elements of $G$ there is a subsequence $\left\{g_{j}^{\prime}\right\}$ such that $\lambda\left(g_{j}^{\prime}\right) u$ converges weakly in $C(G)$, or such that $\lambda\left(g_{j}^{\prime}\right) u$ converges pointwise on $\beta(G)$. This and other results on weakly almost periodic functions are summarized in [1]. The space $W(G)$ of weakly almost periodic functions in $C(G)$ is given the uniform norm.

The Fourier-Stieltjes algebra $B(G)$ of $G$ is the algebra of coordinate functions

$$
u: g \longrightarrow\langle\pi(g) \xi, \eta\rangle
$$$$
\xi, \eta \in \mathscr{K}_{\pi}
$$

of continuous unitary representations $\pi$ of $G$ on Hilbert spaces $\mathscr{C}_{\pi}$; $B(G)$ is normed thus:

$$
\|u\|_{B}=\min \left\{\|\xi\|_{\pi_{\pi}} \cdot\|\eta\|_{\pi_{\pi}}: u=\langle\pi \xi, \eta\rangle\right\} .
$$

The basic facts about $B(G)$ can be found in [4]. We recall from [1] that 


$$
B(G) \subseteq W(G) \subseteq C_{u}(G)
$$

The spaces $B(G), W(G)$, and $C_{u}(G)$ have many similar properties. For example, both left and right translations act continuously and isometrically on each space, their functorial properties are similar, and they are all stable under continuous automorphisms of $G([1,1.8]$, $[4,2.20]$, [8, 34.48]). We shall find more examples below.

We let $E(G)$ denote one of $C_{u}(G), W(G)$ or $B(G)$. If $H$ is a closed subgroup of $G$, then the space of restrictions $\left.E(G)\right|_{H}$ is a subspace of $E(H)$. We are interested in knowing which $E(H)$-functions have extensions in $E(G)$. It is known that $\left.E(G)\right|_{H}=E(H)$ when $G$ is abelian ([1,3.16], [8,34.48]), but this is generally false for nonabelian G. A. Douady observed that if $H$ is a closed normal abelian subgroup of a locally compact group $G$ and $\chi$ is a continuous character of $H$ such that, for any neighborhood $\mathscr{C}$ of the identity $e$ in $G$, there are $s$ in $\mathscr{U}$ and $h$ in $H$ such that $\chi\left(s^{-1} h s\right) \neq \chi(h)$, then $\chi$ cannot be extended to a function $u$ in $B(G)$ (quoted in [5, p. 204]; see also [8, 34.28]). Applying this observation to the semidirect product group $G=T \times_{s} C$, where the torus $T$ acts on the complex numbers $C$ by multiplication, we conclude that $\left.B(G)\right|_{c} \neq B(C)$. Ching Chou [2, p. 192] showed that $\left.W(G)\right|_{C} \neq W(C)$; his example shows also that $\left.C_{u}(G)\right|_{C} \neq$ $C_{u}(\boldsymbol{C})$.

For an arbitrary locally compact group $G$ and closed subgroup $H$ it is known that $\left.B(G)\right|_{H}=B(H)$ if $H$ is open or compact or the connected component of the identity $G_{0}$ of $G$ or the center of $G$ ([10, Prop. 1.1], [11, §2]); $\left.W(G)\right|_{H}=W(H)$ if $H$ is open ([1, 3.14]normality is not needed) or compact or $G_{0}$ (analogous to the $B(G)$ proof); $\left.C_{u}(G)\right|_{H}=C_{u}(H)$ if $H$ is open or compact or $G_{0}$.

In this paper the space $\left.E(G)\right|_{H}$ is characterized when $H$ is a closed normal subgroup of $G$. Further, it is shown that $\left.E(G)\right|_{H}=E(H)$ for any closed subgroup of a [SIN]-group $G$, but that this is false if $G$ belongs to the larger class of [IN]-groups.

1. The extension theorems. Let $N$ be a closed normal subgroup of $G$. We write $h^{g}$ for the conjugate $g^{-1} h g(g, h \in G)$. Functions on $G$ will be denoted by $u, v$, and $w$, and those on $N$ by $x, y$, and $z$. Given such functions $u$ and $x$, we define $u^{g}$ and $x^{g}$ by the formulae:

$$
\begin{aligned}
& u^{g}(h)=u\left(h^{g}\right) \\
& g, h \in G \\
& x^{g}(n)=x\left(n^{g}\right) \\
& g \in G, n \in N \text {. }
\end{aligned}
$$

The maps $u \rightarrow u^{g}$ and $x \rightarrow x^{g}$ are isometries of $E(G)$ and $E(N)$.

THEOREM 1. Let $N$ be a closed normal subgroup of the locally compact group $G$. Then 


$$
\left.E(G)\right|_{N}=\left\{x \in E(N):\left\|x^{g}-x\right\|_{E} \longrightarrow 0 \text { as } g \longrightarrow e\right\},
$$

and, if $\left.x \in E(G)\right|_{N}$, then

$$
\|x\|_{E}=\inf \left\{\|u\|_{E}: u \in E(G),\left.u\right|_{N}=x\right\} .
$$

Proof. We have already remarked that $\left.E(G)\right|_{N} \cong E(N)$; it is further true that

$$
\left\|\left.u\right|_{N}\right\|_{E} \leqq\|u\|_{E}
$$

If $u$ is in $E(G)$, so is $u^{g}$, and as $g$ tends to $e$ in $G$,

$$
\left\|u^{g}-u\right\|_{E} \longrightarrow 0 \text {. }
$$

Consequently

$$
\left.E(G)\right|_{N} \subseteq\left\{x \in E(N):\left\|x^{g}-x\right\|_{E} \longrightarrow 0 \text { as } g \longrightarrow e\right\} .
$$

We remark at this point that if $\chi$ is a continuous character of $N$ and belongs to $\left.E(G)\right|_{N}$ there must be a neighborhood $\mathscr{C}$ of $e$ in $G$ such that, if $g$ belongs to $\mathscr{C}$, then

$$
\left\|\chi^{g}-\chi\right\|_{E}<1 \text {. }
$$

It then follows easily that $\chi^{g}=\chi$ for $g$ in $\mathscr{\ell}$. This is a proof of Douady's observation quoted in the introduction.

To complete the proof of the theorem is less trivial. As in the proof of the open mapping theorem, it suffices to show that, for any $x$ in $E(N)$ satisfying $\left\|x^{g}-x\right\|_{E} \rightarrow 0$ as $g \rightarrow e$, and for any small positive $\varepsilon$, there is some $u$ in $E(G)$ for which

$$
\|u\|_{E}=\|x\|_{E}
$$

and

$$
\left\|\left.u\right|_{N}-x\right\|_{E}<\varepsilon .
$$

Given such an $x$ and $\varepsilon$, we can find neighborhoods $\mathscr{U}$ of $e$ in $G$ and $\mathscr{P}$ of $e$ in $N$ such that

$$
\begin{array}{cr}
\left\|x^{g}-x\right\|_{E}<\varepsilon / 2 & g \in \mathscr{U} \\
\left\|\lambda\left(n^{-1}\right) x-x\right\|_{E}<\varepsilon / 2 & n \in \mathscr{X} .
\end{array}
$$

Let $\mathscr{V}$ be a compact neighborhood of $e$ in $G$ such that

$$
\begin{gathered}
\mathscr{Y} \leqq \mathscr{U} \\
\mathscr{V}^{-1} \cdot \mathscr{V} \cap N \subseteq \mathscr{X} .
\end{gathered}
$$

Take a nonnegative continuous function $v$ on $G$ such that 


$$
\begin{aligned}
\operatorname{supp}(v) \cong \mathscr{Y} \\
1=\int_{G / N} d \dot{g}\left[\int_{N} d n v(g n)\right]^{2} \\
=\int_{G / N} d \dot{g} \int_{N} d n^{\prime} v\left(g n^{\prime}\right) \int_{N} d n v(g n) \\
=\int_{G / N} d \dot{g} \int_{N} d n^{\prime} v\left(g n^{\prime}\right) \int_{N} d n v\left(g n^{\prime} n\right) \\
=\int_{G} d g \int_{N} d n v(g) v(g n) ;
\end{aligned}
$$

here we have assumed that the Haar measure $d g, d \dot{g}$, and $d n$ of $G$, $G / N$ and $N$ are normalized so that

$$
\int_{G / N} d \dot{g} \int_{N} d n w(g n)=\int_{G} d g w(g),
$$

$\left(C_{c}(G)\right.$ the space of compactly supported functions) ([8, 28.54]). We now define the function $u$ on $G$ by the formula

$$
u\left(g^{\prime}\right)=\int_{G} d g \int_{N} d n v\left(g^{\prime} g\right) v(g n) x(n), \quad g^{\prime} \in G .
$$

This is a variation of a device of H. Reiter [13], who considered functions $w$ of the form

$$
w\left(g^{\prime}\right)=\int_{N} d n v\left(g^{\prime} n\right) x\left(n^{-1}\right), \quad \quad g^{\prime} \in G .
$$

We shall check later that $u$ belongs to $E(G)$ and that $\|u\|_{E} \leqq\|x\|_{E}$. For the moment we obtain an expression for $\left.u\right|_{N}$ and estimate $\left\|\left.u\right|_{N}-x\right\|_{E}$.

For any $n^{\prime}$ in $N$,

$$
\begin{aligned}
u\left(n^{\prime}\right) & =\int_{G} d g \int_{N} d n v(g) v\left(n^{\prime-1} g n\right) x(n) \\
& =\int_{G} d g \int_{N} d n v(g) v\left(g\left(g^{-1} n^{\prime-1} g\right) n\right) x(n) \\
& =\int_{G} d g \int_{N} d n v(g) v(g n)\left[\lambda\left(n^{-1}\right) x\right]^{g n}\left(n^{\prime}\right) .
\end{aligned}
$$

The map from $G \times N$ to $E(N)$ defined by the rule

$$
(g, n) \longrightarrow v(g) v(g n)\left[\lambda\left(n^{-1}\right) x\right]^{g n}
$$

is continuous and compactly supported, so the vector-valued Riemann integral

$$
\int_{G} d g \int_{N} d n v(g) v(g n)\left[\lambda\left(n^{-1}\right) x\right]^{g n}
$$


exists, and, by the pointwise equality (7), is equal to $\left.u\right|_{N}$. We note from (6) that

$$
x=\int_{G} d g \int_{N} d n v(g) v(g n) x,
$$

so that

$$
\begin{aligned}
\left\|\left.u\right|_{N}-x\right\|_{E} & \leqq \int_{G} d g \int_{N} d n v(g) v(g n)\left\|\left[\lambda\left(n^{-1}\right) x\right]^{g n}-x\right\|_{E} \\
& \leqq \int_{G} d g \int_{N} d n v(g) v(g n)\left(\left\|\left[\lambda\left(n^{-1}\right) x\right]^{g n}-x^{g n}\right\|_{E}+\left\|x^{g n}-x\right\|_{E}\right) \\
& =\int_{G} d g \int_{N} d n v(g) v(g n)\left(\left\|\lambda\left(n^{-1}\right) x-x\right\|_{E}+\left\|x^{g n}-x\right\|_{E}\right) .
\end{aligned}
$$

If $v(g) \cdot v(g n)$ is nonzero, then from (5), $g$ and $g n$ are in $\mathscr{T}$; from (1) and (3), $\left\|x^{g n}-x\right\|_{E}<\varepsilon / 2$ and from (2) and (4), $\left\|\lambda\left(n^{-1}\right) x-x\right\|_{E}<\varepsilon / 2$. By applying (6) again, we conclude that

$$
\left\|\left.u\right|_{N}-x\right\|_{E} \leqq \varepsilon \text {. }
$$

It remains to show that $u$ belongs to $E(G)$ and that $\|u\|_{E} \leqq\|v\|_{E}$. The easiest case is where $E=C_{u}$; we leave this verification to the reader. Let us consider the case where $E=B$. Then $x$ can be written in the form

$$
x(n)=\langle\sigma(n) \xi, \eta\rangle,
$$

$n \in N$,

where $\sigma$ is a unitary representation of $N$ on a Hilbert space $\mathscr{H}_{\sigma}$, $\xi$ and $\eta$ belong to $\mathscr{C}_{\sigma}$, and $\|v\|_{B}=\|\xi\|_{\mathscr{C}_{\sigma}} \cdot\|\eta\|_{\mathscr{C}_{\sigma}} \cdot$ Now, for any $g^{\prime}$ in $G$,

$$
\begin{aligned}
u\left(g^{\prime}\right) & =\int_{G} d g \int_{N} d n v(g) v\left(g^{\prime-1} g n\right) x(n) \\
& =\int_{G / N} d \dot{g} \int_{N} d n^{\prime} \int_{N} d n v\left(g n^{\prime}\right) v\left(g^{\prime-1} g n^{\prime} n\right)\langle\sigma(n) \xi, \eta\rangle \\
& =\int_{G / N} d \dot{g} \int_{N} d n^{\prime} \int d n v\left(g^{\prime-1} g n\right) v\left(g n^{\prime}\right)\left\langle\sigma(n) \xi, \sigma\left(n^{\prime}\right) \eta\right\rangle \\
& =\int_{G / N} d \dot{g}\left\langle\int_{N} d n v\left(g^{\prime-1} g n\right) \sigma(n) \xi, \int_{N} d n^{\prime} v\left(g n^{\prime}\right) \sigma\left(n^{\prime}\right) \eta\right\rangle,
\end{aligned}
$$

which the reader will recognize as a coordinate function of the representation $\pi$ of $G$ unitarily induced from $\sigma$ (see [5, VI. 2]). Further,

$$
\|u\|_{B} \leqq\left\|\int_{N} d n v(g n) \sigma(n) \xi\right\|_{\mathscr{C}_{\pi}} \cdot\left\|\int_{N} d n v(g n) \sigma(n) \eta\right\|_{\mathscr{C}_{\pi}},
$$

and

$$
\left\|\int_{N} d n v(g n) \sigma(n) \zeta\right\|_{\mathscr{C}_{\pi}}=\left(\int_{G / N} d \dot{g}\left[\left\|\int_{N} d n v(g n) \sigma(n) \zeta\right\|_{\mathscr{C}_{0}}\right]^{2}\right)^{1 / 2} \leqq\|\zeta\|_{\mathscr{C}_{0}}
$$


from (6), whence $\|u\|_{B} \leqq\|x\|_{B}$.

The other case to consider is where $E$ is $W$. By repeating the argument used to deduce (8) from (7), we deduce that, for fixed $g^{\prime}$ in $G$,

$$
\left.\lambda\left(g^{\prime}\right) u\right|_{N}=\int_{G} d g \int_{N} d n v\left(g^{\prime-1} g\right) v(g n) \lambda\left(n^{-1}\right) x^{g n},
$$

as a vector-valued integral, from which we see that $\left.\lambda\left(g^{\prime}\right) u\right|_{N}$ lies in $W(N)$ for each $g^{\prime}$ in $G$. The function $u$ is uniformly continuous and supported by $\mathscr{V}^{-1} \mathscr{C} N$, where $\mathscr{V}^{-1} \mathscr{V}$ is a compact subset of $G$. It is trivial to show that $\|u\|_{\infty} \leqq\|x\|_{\infty}$, so application of the following lemma completes the proof of the theorem.

Lemma. Suppose that $u$ belongs to $C_{u}(G)$ and that

(i) there is a compact subset $K$ of $G$ such that $\operatorname{supp}(u) \subseteq K N$,

(ii) $\left.\lambda(g) u\right|_{N}$ belongs to $W(N)$ for all $g$ in $G$. Then $u$ is in $W(G)$.

Proof. Let $\left\{g_{j}\right\}$ be a sequence of elements of $G$. We want to show that $\left\{\lambda\left(g_{j}\right) u\right\}$ has a weakly convergent subsequence in $C(G)$. We let $p: G \rightarrow G / N$ be the canonical projection.

Case (a). $\left\{p\left(g_{j}\right)\right\}$ is unbounded in $G / N$.

In this case there is a subsequence $\left\{g_{j}^{\prime}\right\}$ such that $\left\{p\left(g_{j}^{\prime}\right)\right\}$ eventually avoids any compact subset of $G / N$. Let $s$ be a nonnegative compactly supported function on $G / N$ such that

$$
|u(g)| \leqq s \circ p(g), \quad g \in G .
$$

Then

$$
\left|\lambda\left(g_{j}^{\prime}\right) u\right| \leqq\left[\lambda\left(p\left(g_{j}^{\prime}\right)\right) s\right] \circ p .
$$

Now $\left\{\lambda\left(p\left(g_{j}^{\prime}\right)\right) s\right\}$ converges to zero pointwise on $\beta(G / N)$. The map $s \rightarrow s \circ p$ injects $C(G / N)$ into $C(G)$, and so dually projects $\beta(G)$ into $\beta(G / N)$. It follows that $\left\{\lambda\left(p\left(g_{j}^{\prime}\right)\right) s \circ p\right\}$ converges to zero pointwise on $\beta(G)$; a fortiori $\left\{\lambda\left(g_{j}^{\prime}\right) u\right\}$ converges pointwise to zero on $\beta(G)$, so converges weakly to zero in $C(G)$.

Case (b). $\left\{p\left(g_{j}\right)\right\}$ is bounded in $G / N$.

There is a compact set $K^{\prime}$ in $G$ such that each $g_{j}$ can be expressed, not necessarily uniquely, as $n_{j} k_{j}$, with $n_{j}$ in $N$ and $k_{j}$ in $K^{\prime}$. Since $K^{\prime}$ is compact, there is a subsequence $\left\{g_{j}^{\prime}\right\}$ of $\left\{g_{j}\right\}$ such that the corresponding sequence $\left\{k_{j}^{\prime}\right\}$ has a limit point $k_{0}$, say. Note that

$$
\left\|\lambda\left(n_{j}^{\prime} k_{j}^{\prime}\right) u-\lambda\left(n_{j}^{\prime} k_{0}\right) u\right\|_{\infty}=\left\|\lambda\left(k_{j}^{\prime}\right) u-\lambda\left(k_{0}\right) u\right\|_{\infty} \longrightarrow 0
$$


as $j \rightarrow \infty$. Hence, to show that $\left\{\lambda\left(g_{j}^{\prime}\right) u\right\}$ has a weakly convergent subsequence, it suffices to show that $\left\{\lambda\left(n_{j}^{\prime} k_{0}\right) u\right\}$ has one; it certainly suffices to show that the set $\lambda\left(N k_{0}\right) u$ is relatively weakly compact.

We write $C^{0}(G)$ for the subspace of $C_{u}(G)$ of functions vanishing off $K^{\prime} K N$. The Hahn-Banach theorem implies that the weak topology of $C^{0}(G)$ coincides with its relative weak topology as a subspace of $C(G)$. The function $u$ lies in $C^{0}(G)$, as do the translates $\lambda\left(n_{j}^{\prime} k_{0}\right) u$.

Next we denote by $C\left(K^{\prime} K ; C_{u}(N)\right)$ the space of $C_{u}(N)$-valued continuous functions on $K^{\prime} K$, and observe that $\sigma: C^{0}(G) \rightarrow C\left(K^{\prime} K\right.$; $\left.C_{u}(N)\right)$, defined by setting

$$
[\sigma w(k)](n)=w(n k) \quad k \in K^{\prime} K, n \in N,
$$

is an isometric embedding of $C^{0}(G)$ into $C\left(K^{\prime} K ; C_{u}(N)\right)$; the weak topology on $C^{0}(G)$ and the relative weak topology on $\sigma\left(C^{0}(G)\right)$ therefore coincide. Further, if $w$ satisfies the hypotheses of the lemma, then $w$ is in $C\left(K^{\prime} K ; W(N)\right)$. We denote by $\Lambda$ the strongly continuous isometric representation of $N$ on $C\left(K^{\prime} K ; C_{u}(N)\right)$ by the formula

$$
[\Lambda(n) t](k)\left(n^{\prime}\right)=t(k)\left(n^{-1} n^{\prime}\right) \quad k \in K^{\prime} K, n, n^{\prime} \in N .
$$

Then

$$
\begin{aligned}
{[\Lambda(n) \sigma w](k)\left(n^{\prime}\right) } & =\sigma w(k)\left(n^{-1} n^{\prime}\right) \\
& =w\left(n^{-1} n^{\prime} k\right) \\
& =\lambda(n) w\left(n^{\prime} k\right) \\
& =[\sigma \lambda(n) w](k)\left(n^{\prime}\right),
\end{aligned}
$$

so that, to prove the lemma, it will suffice to establish the following claim.

Claim. If $t$ is in $C\left(K^{\prime} K ; W(N)\right)$, then the set $\Lambda(N) t$ is relatively weakly compact.

To prove the claim, we consider first functions of the form $w \otimes y$ :

$$
w \otimes y:(k, n) \longrightarrow w(k) y(n) . \quad k \in K^{\prime} K, n \in N,
$$

where $w$ and $y$ are in $C\left(K^{\prime} K\right)$ and $W(N)$ respectively. Clearly

$$
\Lambda(n) w \otimes y=w \otimes \lambda(n) y \text {. }
$$

Now suppose that $\Phi$ is a functional on $C\left(K^{\prime} K ; C_{u}(N)\right)$. Then $\Phi$ gives rise to a functional $\Phi_{w}$ on $C_{u}(N)$ by the formula

$$
\left\langle\Phi_{w}, y\right\rangle=\langle\Phi, w \otimes y\rangle \text {. }
$$

Since $y$ is in $W(N)$, any sequence $\left\{\lambda\left(n_{j}\right) y\right\}$ has a weakly convergent subsequence $\left\{\lambda\left(n_{j}^{\prime}\right) y\right\}$; thus the sequence $\left\{\Lambda\left(n_{j}\right) w \otimes y\right\}$ has a weakly convergent subsequence $\left\{\Lambda\left(n_{j}^{\prime}\right) w \otimes y\right\}$. We conclude that $\Lambda(N) w \otimes y$ is relatively weakly compact. 
Now because $K^{\prime} K$ is compact, any function in $C\left(K^{\prime} K ; W(N)\right)$ can be approximated in norm by finite sums of functions of the form just considered. The set of functions $t$ in $C\left(K^{\prime} K ; W(N)\right)$ for which the set $\Lambda(N) t$ is relatively weakly compact is a closed linear subspace of $C\left(K^{\prime} K ; W(N)\right)$ (c.f., the proof that $W(G)$ is a closed subspace of $\left.C_{u}(G)[1,1.6]\right)$. This establishes the claim and completes the proof of the lemma.

A locally compact group $G$ is said to have small invariant neighborhoods (written $G \in[\mathrm{SIN}]$ ) when there is a basis of neighborhoods of $e$ in $G$-invariant under inner automorphisms. A function $v$ on $G$ is called central if $v\left(g g^{\prime}\right)=v\left(g^{\prime} g\right)$ for all $g$ and $g^{\prime}$ in $G$. A [SIN]-group has nonnegative continuous central functions $v$ with arbitrarily small supports. Note that

$$
\int v(g) d g=\int v\left(g^{\prime} g\right) d g=\int v\left(g g^{\prime}\right) d g=\Delta\left(g^{\prime}\right) \int v(g) d g,
$$

so [SIN]-groups are unimodular. A convenient reference for results on [SIN]-groups is the monograph of S. Grosser and M. Moskovitz [6].

THEOREM 2. Suppose that $H$ is a closed subgroup of the [SIN]group G. Then

$$
\left.E(G)\right|_{H}=E(H)
$$

and, if $x$ is in $E(H)$,

$$
\|x\|_{E}=\inf \left\{\|u\|_{E}: u \in E(G),\left.u\right|_{I I}=x\right\} .
$$

Proof. As before, it suffice to show that, for any $x$ in $E(H)$ and small positive $\varepsilon$, there exists $u$ in $E(G)$ such that

$$
\begin{aligned}
& \|u\|_{E} \leqq\|x\|_{E} \\
& \left\|\left.u\right|_{H}-x\right\|_{E}<\varepsilon .
\end{aligned}
$$

Since $G$ has small invariant neighborhoods, so does $H$. Then both $G$ and $H$ are unimodular, so there exists a $G$-invariant measure $d \dot{g}$ on the quotient space $G / H$ (see $[8,15.24]$ ); we assume that the Haar measures of $G$ and $H$ are adjusted so that

$$
\int_{G} d g w(g)=\int_{G / H} d g \int_{H} d h w(g h) \quad w \in C_{c}(G) .
$$

Let $V$ be a compact neighborhood of $e$ in $G$ so that

$$
\left\|\lambda\left(h^{-1}\right) x-x\right\|_{E}<\varepsilon \quad h \in V^{-1} V \cap H,
$$


and let $v$ be a nonnegative continuous central function on $G$ such that

$$
\begin{gathered}
\operatorname{supp}(v) \leqq V \\
\int_{G / I I} d \dot{g} \int_{I I} d h v(g h)^{2}=1 .
\end{gathered}
$$

We define the function $u$ on $G$ by the formula

$$
u\left(g^{\prime}\right)=\int_{G} d g \int_{I I} d h v\left(g^{\prime} g\right) v(g h) x(h) \quad \quad g^{\prime} \in G .
$$

For any $h^{\prime}$ in $H$,

$$
\begin{aligned}
u\left(h^{\prime}\right) & =\int_{G} d g \int_{I} d h v(g) v\left(h^{\prime-1} g h\right) x(h) \\
& =\int_{G} d g \int_{I I} d h v(g) v\left(g h h^{\prime-1}\right) x(h) \\
& =\int_{G} d g \int_{I I} d h v(g) v(g h)\left[\lambda\left(h^{-1}\right) x\right]\left(h^{\prime}\right),
\end{aligned}
$$

since $v$ is central and $H$ is unimodular. This pointwise equality is very similar to the equality (7) of Theorem 1. By repeating the argument of Theorem 1, and using (9), (10), and (11), we deduce that

$$
\left\|\left.u\right|_{H I}-x\right\|_{E}<\varepsilon \text {. }
$$

The proof that $u$ is in $E(G)$ if $E$ is $C_{u}$ is easy, and if $E$ is $B$, is identical to the corresponding part of the proof of Theorem 1 . We shall therefore examine the $W$-case only. We define $v(x)$ by the rule

$$
v(x)(g)=\int_{H I} d h v\left(g^{-1} h\right) x(h)
$$$$
g \in G ;
$$

since $u=v * v(x)$ and $W(G)$ is closed under convolutions by $L^{\mathrm{t}}(G)$ functions, it suffices to show that $v(x)$ is in $W(G)$.

Suppose then that $x$ is in $W(H)$ and that $\left\{g_{j}\right\}$ is a sequence of points of $G$. As before, we let $p: G \rightarrow G / H$ be the canonical projections and examine two cases.

Case (a). $\left\{p\left(g_{j}\right)\right\}$ is unbounded in $G / H$.

We claim that if $\left\{p\left(g_{j}^{\prime}\right)\right\}$ is a subsequence tending to infinity, then $\left\{\lambda\left(g_{j}^{\prime}\right) v(x)\right\}$ converges weakly to zero. First, let $\underline{1}$ be the function on $H$ taking the value one on $H$. Then

$$
\left|\lambda\left(g_{j}^{\prime}\right) v(x)\right| \leqq\|x\|_{\infty} \lambda\left(g_{j}^{\prime}\right) v(\underline{1}),
$$

so it suffice to show that $\left\{\lambda\left(g_{j}^{\prime}\right) v(1)\right\}$ converges to zero. Next, since $v$ is central, 


$$
\begin{aligned}
\lambda\left(g_{j}^{\prime}\right) v(\underline{1})(g) & =\int_{H} d h v\left(g^{-1} g_{j} h\right) \\
& =\int_{H} d h v\left(h g^{-1} g_{j}\right)
\end{aligned}
$$

From the second integral expression, we see that $\lambda\left(g_{j}^{\prime}\right) v(\underline{1})(g h)=$ $\lambda\left(g_{j}^{\prime}\right) v(\underline{1})(g)(g$ in $G, h$ in $H)$, so that $\lambda\left(g_{j}^{\prime}\right) v(\underline{1})$ is of the form $\left[\Lambda\left(g_{j}^{\prime}\right) s\right] \circ p$, for some continuous function $\Lambda\left(g_{j}^{\prime}\right) s$ on $G / H$. Again from the second integral expression, we see that $\lambda\left(g_{j}^{\prime}\right) v(\underline{1})(g)$ is nonzero only if $h g^{-1} g_{j}$ is in the support of $v$ for some $h$ in $H$, i.e., only if $g$ is in $g_{j}^{-1} \operatorname{supp}(v)^{-1} H$. Thus $\left\{\Lambda\left(g_{j}^{\prime}\right) s\right\}$ is a sequence of compactly supported functions on $G / H$. Finally, from the first integral expression, we note that $\lambda\left(g_{j}^{\prime}\right) v(\underline{1})(g)$ is nonzero only if $g^{-1} g_{j} h$ lies in the support of $v$ for some $h$ in $H$, i.e., only if $g_{j}$ lies in the set $g \operatorname{supp}(v) H$. Since $\left\{p\left(g_{j}\right)\right\}$ tends to infinity, $\left\{\lambda\left(g_{j}^{\prime}\right) v(\underline{1})(g)\right\}$ converges to zero.

The sequence $\left\{\Lambda\left(g_{j}^{\prime}\right) s\right\}$ is therefore a sequence of compactly supported function on $G / H$ which converges pointwise to zero. Then $\left\{\Lambda\left(g_{j}^{\prime}\right) s\right\}$ converges pointwise to zero on $\beta(G / H)$, so that $\left\{\left[\Lambda\left(g_{j}^{\prime}\right) s\right] \circ p\right\}$ converges pointwise to zero on $\beta(G)$. In other words, $\left\{\lambda\left(g_{j}^{\prime}\right) v(\underline{1})\right\}$ converges weakly to zero.

Case (b). $\left\{p\left(g_{j}\right)\right\}$ is bounded in $G / H$.

In this case there is a compact set $K$ in $G$ such that each $g_{j}$ can be written in the form $k_{j} h_{j}$, with $k_{j}$ in $K$ and $h_{j}$ in $H$. Note that, for $g$ in $G$,

$$
\begin{aligned}
\lambda\left(g_{j}\right) v(x) & =\int_{H} d h v\left(g^{-1} k_{j} h_{j} h\right) x(h) \\
& =\int_{H} d h v\left(g^{-1} k_{j} h\right) x\left(h_{j}^{-1} h\right) \\
& =\int_{H} d h v\left(h g^{-1} k_{j}\right) \lambda\left(h_{j}\right) x(h) .
\end{aligned}
$$

There is a subsequence $g_{j}^{\prime}$ of $g_{j}$ such that $\left\{v\left(h g^{-1} k_{j}\right)\right\}$ converges to $v\left(h g^{-1} k_{0}\right)$, say, uniformly in $g$ and $h$, and such that $\left\{\lambda\left(h_{j}\right) x\right\}$ converges weakly to $y$, say. It then follows that $\left\{\lambda\left(g_{j}\right) v(x)\right\}$ converges to $\left\{\lambda\left(k_{0}\right) v(y)\right\}:$ first,

$$
\begin{aligned}
& \left|\left[\lambda\left(g_{j}\right) v(x)-\lambda\left(k_{0} h_{j}\right) v(x)\right](g)\right| \\
& \quad \leqq \int_{I I} d h\left|v\left(h g^{-1} k_{j}\right)-v\left(h g^{-1} k_{0}\right)\right|\left\|\lambda\left(h_{j}\right) x\right\|_{\infty}
\end{aligned}
$$

which converges to zero uniformly on $G$. Second,

$$
\lambda\left(k_{0} h_{j}\right) v(x)-\lambda\left(k_{0}\right) v(y)=\lambda\left(k_{0}\right) v\left(\lambda\left(h_{j}\right) x-y\right) \text {. }
$$

Given a linear functional $\Phi$ on $C(G)$, then $\Phi^{\prime}$, defined 


$$
\left\langle\Phi^{\prime}, z\right\rangle=\left\langle\Phi, \lambda\left(k_{0}\right) v(z)\right\rangle
$$

is a linear functional on $C(H)$. Since $\left\{\left\langle\Phi^{\prime}, \lambda\left(h_{j}\right) x-y\right\rangle\right\}$ converges to zero, $\left\{\left\langle\Phi, \lambda\left(k_{0}\right) v\left(\lambda\left(h_{j}\right) x-y\right)\right\rangle\right\}$ does also, and $\left\{\lambda\left(k_{0}\right) v\left(\lambda\left(h_{j}\right) x-y\right)\right\}$ converges weakly to zero.

2. Corollaries. Hereafter, $K$ denotes a compact group. We recall that the radical of a connected Lie group is the maximal connected solvable normal subgroup.

COROLlary 1. If $G$ is a connected Lie group, the following properties are equivalent:

(i) for every closed normal subgroup $N$ of $G,\left.E(G)\right|_{N}=E(N)$.

(ii) the radical $R$ of $G$ is central.

Proof. (i) $\Rightarrow$ (ii). The following claim is the key to this step: if $A$ is a connected closed normal abelian subgroup of a connected Lie group $H$ and $\left.E(H)\right|_{A}=E(A)$, then $A$ is central in $H$.

To establish the claim, we note that $A$ can be written in the form $R^{b} \times T^{c}, T$ being the torus, that there exist $2 b+c$ characters $\chi_{j}$ of $R^{b} \times T^{c}$ such that

$$
\cap \operatorname{ker} \chi_{j}=\{e\}
$$

and that there is an open set $U$ in $H$ such that

$$
\chi_{j}\left(h^{-1} a h\right)=\chi_{j}(a) \quad j=1, \cdots, 2 b+c
$$

by Douady's observation, cited in the introduction. Since $H$ is connected we conclude that $A$ is central, as claimed.

We now define inductively the solvable series of the radical $R$ of $G$ :

$$
R_{0}=R, R_{j+1}=\left[R_{j}, R_{j}\right] \quad j=0,1, \cdots .
$$

This series terminates:

$$
R=R_{0} \triangleright R_{1} \triangleright \cdots \triangleright R_{k-1} \triangleright R_{k}=\{e\} ;
$$

$R_{j} / R_{j+1}$ is a nontrivial connected abelian Lie group and the $R_{j}$ are characteristic in $G$.

We suppose temporarily that $k \geqq 2$, i.e., that $R$ is not abelian, and derive a contradiction. The hypothesis (i) implies that $\left.E(G)\right|_{R_{k-1}}=$ $E\left(R_{k-1}\right)$, so that $R_{k-1}$ is central in $G$. Further,

$$
\begin{aligned}
\left.E\left(G / R_{k-1}\right)\right|_{R_{k-2} / R_{k-1}} & \left.\cong E_{R_{k-1}}(G)\right|_{R_{k-2}} \\
& =E_{R_{k-1}}\left(R_{k-2}\right) \\
& \cong E\left(R_{k-2} / R_{k-1}\right),
\end{aligned}
$$


where, for example, $E_{R_{k-1}}(G)$ is the space of $E(G)$-functions constant on cosets of $R_{k-1}$ in $G$. So $R_{k-2} / R_{k-1}$ is central in $G / R_{k-1}$. However, $R_{k-2}$ is not abelian, so contains a noncentral one-parameter subgroup $A$. Since $R_{k-1}$ is central, $A \$ R_{k-1}$, and $A R_{k-1} / R_{k-1}$ is a one-parameter subgroup of $G / R_{k-1}$. Since $R_{k-2} / R_{k-1}$ is central in $G / R_{k-1}, A R_{k-1} / R_{k-1}$ is certainly normal in $G / R_{k-1}$, whence $A R_{k-1}$ is normal in $G$. Finally, $R_{k-1}$ is central in $G$ and $A$ is abelian but not central, so that $A R_{k-1}$ is abelian but not central. We have just produced a noncentral normal abelian subgroup of $G$, which, by the claim, contradicts hypothesis (i). We conclude that $k \leqq 1$, i.e., that $R$ is abelian. Further, $\left.E(G)\right|_{R}=E(R)$, whence $R$ is central by the claim.

(ii) $\Rightarrow$ (i). Let $\widetilde{G}$ be the universal covering group of $G$, and $p$ be the canonical projection of $\widetilde{G}$ onto $G$. Standard results of Lie theory imply that $\widetilde{G}$ can be written as a direct product

$$
S_{1} \times \cdots \times S_{n} \times A,
$$

where the $S_{j}$ are simple (in the Lie algebra sense) and $A$ is abelian (see, for example, [7], [9]).

If $N$ is a closed normal subgroup of $G$, then $p^{-1}(N)$ is a closed normal subgroup of $\widetilde{G}$. Suppose that $\left(s_{1}, \cdots, s_{n}, a\right)$ is an element of $p^{-1}(N)$. Then $\left(e, \cdots, e, t_{j}, e, \cdots, e, e\right)$ normalizes $p^{-1}(N)$, so that

$$
\left(s_{1}, \cdots, s_{j-1}, t_{j}^{-1} s_{j} t_{j}, s_{j+1}, \cdots, s_{n}, a\right) \in p^{-1}(N)
$$

whence $\left(e, \cdots, e, s_{j}^{-1} t_{j}^{-1} s_{j} t_{j}, e, \cdots, e, e\right) \in p^{-1}(N)$. It follows that $s_{j}$ lies in the discrete center of $S_{j}$ or $S_{j}$ is contained in $p^{-1}(N)$.

Now let $U$ be a small neighborhood of $e$ in $\widetilde{G}$ of the form $U_{1} \times \cdots \times U_{n} \times U_{0}$ such that $p$, restricted to $U$, is a local isomorphism. We define a continuous projection $q$ of $U$ into $U \cap p^{-1}(N)$ by the rule

$$
q\left(t_{1}, \cdots, t_{n}, b\right)=\left(t_{1}^{\prime}, \cdots, t_{n}^{\prime}, e\right),
$$

where $t_{j}^{\prime}=t_{j}$ if $S_{j}$ is contained in $p^{-1}(N)$ and $t_{j}^{\prime}=e$ otherwise. We observe that, for $\left(t_{1}, \cdots, t_{n}, b\right)$ in $U$ and $\left(s_{1}, \cdots, s_{n}, a\right)$ in $p^{-1}(N)$,

$$
\begin{aligned}
& \left(t_{1}, \cdots, t_{n}, b\right)^{-1}\left(s_{1}, \cdots, s_{n}, a\right)\left(t_{1}, \cdots, t_{n}, b\right) \\
& \quad=q\left(t_{1}, \cdots, t_{n}, b\right)^{-1}\left(s_{1}, \cdots, s_{n}, a\right) q\left(t_{1}, \cdots, t_{n}, b\right),
\end{aligned}
$$

since $t_{j}^{\prime}$ is exactly $t_{j}$ unless $s_{j}$ is central.

We now pass to the quotient group $G$, where we have a continuous projection, again called $q$, of $p(U)$ into $p(U) \cap N$ such that

$$
g^{-1} n g=q g^{-1} n q g \quad g \in p(U), n \in N .
$$

If $x$ is in $E(N)$ and $g$ is in $p(U), x^{g}=x^{q g}$. Thus 


$$
\left\|x^{g}-x\right\|_{E}=\left\|x^{q g}-x\right\|_{E} \cdot
$$

As $g \rightarrow e$ in $p(U), q g \rightarrow e$ in $p(U) \cap N$, so $\left\|x^{g}-x\right\|_{E} \rightarrow 0$; by Theorem $1, E(N)=\left.E(G)\right|_{N}$, as required.

CoROLlaRY 2. If $G$ is a connected Lie group, the following properties are equivalent:

(i) for every closed subgroup $H$ of $G,\left.E(G)\right|_{H}=E(H)$.

(ii) $G$ is of the form $\boldsymbol{R}^{b} \times K$.

Proof. (ii) $\Rightarrow$ (i). A group of the form $\boldsymbol{R}^{b} \times K$ has small invariant neighborhoods, so Theorem 2 applies.

(i) $\Rightarrow$ (ii). Assuming that (i) holds, the first condition of Corollary 1 is satisfied, so the radical $R$ of $G$ is central, and $G / R$ is semisimple. If $G / R$ is noncompact, it has an Iwasawa decomposition KAN, where $K$ is compact, $A$ is abelian, and $N$ is nilpotent. The group $A$ normalizes but does not centralize $N$. For details, see [7, Chap. VI]. Let $G^{\prime}$ be the solvable subgroup of $G$ such that $G^{\prime} / R=A N$. If $H$ is a closed normal subgroup of $G^{\prime}$, then

$$
\left.E\left(G^{\prime}\right)\right|_{H}=\left.\left.E(G)\right|_{G^{\prime}}\right|_{H}=\left.E(G)\right|_{H}=E(H),
$$

so that $G^{\prime}$ is a solvable group satisfying the condition of Corollary 1 , and therefore $G^{\prime}$ is abelian. But then the quotient group $G^{\prime} / R$ is abelian, a contradiction. Thus $G / R$ must be compact. Structure theory of such Lie groups now implies the required result ([6, Th. 4.3]).

3. Examples. The following are examples of groups $G$ with normal abelian subgroups $N$ for which $E(N) \neq\left. E(G)\right|_{N}$. All are semidirect products of the form $G=H \times_{s} N$. To find an example of an $E(N)$-function with no extension in $E(G)$, is suffices to find a character $\chi$ of $N$ such that $\chi^{h} \neq \chi$ for small nonidentical $h$ in $H$. Alternatively, Chou's construction can be used to provide examples of $W(N)$-functions with no uniformly continuous extensions.

Example 1. The " $a x+b$ " group. Take $G$ to be the semidirect product $\boldsymbol{R}_{+}^{*} \boldsymbol{X}_{s} \boldsymbol{R}$, where the multiplicative group of positive integers acts on $\boldsymbol{R}$ by multiplication, and $N$ to be $\boldsymbol{R}$.

Example 2. The Heisenberg group. Let $G$ be $R \times{ }_{s} \boldsymbol{R}^{2}$, where $\boldsymbol{R}$ acts on $\boldsymbol{R}^{2}$ by the rule $a(b, c)=(b, c+a b)$, and let $N$ be $\boldsymbol{R}^{2}$.

Example 3. Let $G$ be $T \times_{s} C$, where the torus acts on $C$ by multiplication, and let $N$ be $\boldsymbol{C}$. 
Example 4. If $G$ is the Heisenberg group and $D$ the central discrete subgroup of elements $(0,(0, n))(n \in Z)$, then $G / D$ is a connected Lie group with compact neighborhoods of the identity invariant under inner automorphisms, but is not a [SIN]-group. Extension from the normal abelian subgroup $R^{2} / D$ is generally not possible.

We conclude with a rather different example.

Example 5. SL $(2, \boldsymbol{R})$. For the group $\mathrm{SL}(2, \boldsymbol{R})$, it can be shown that any $B$-function can be written as the sum of a constant function and a function which vanishes at infinity (see [3]). The group $\mathrm{SL}(2, \boldsymbol{R})$ contains many one-parameter subgroups $H$ which are noncompact; for such subgroups, the restriction space $\left.B(G)\right|_{H}$ is much smaller than $B(H)$.

\section{REFERENCES}

1. R. B. Burckel, Weakly Almost Periodic Functions on Semigroups, Gordon and Breach, New York, 1970.

2. C. Chou, Weakly almost periodic functions and almost convergent functions on a group, Trans. Amer. Math. Soc., 206 (1975), 175-200.

3. M. Cowling, The Fourier-Stieltjes algebra of a semisimple group, to appear in Colloq. Math.

4. P. Eymard, L'algèbre de Fourier d'un groupe localement compact, Bull. Soc. Math. de France, 92 (1964), 181-236.

5. S. Gaal, Linear Analysis and Representation Theory, Springer-Verlag, Berlin, Heidelberg, New York, 1973.

6. S. Grosser and M. Moskovitz, On central topological groups, Trans. Amer. Math. Soc., 127 (1967), 317-340.

7. S. Helgason, Differential Geometry and Symmetric Spaces, Academic Press, New York, 1972.

8. E. Hewitt and K. A. Ross, Abstract Harmonic Analysis, Vols I, II, Springer-Verlag, Berlin, Heidelberg, New York, 1963, 1970.

9. G. Hochschild, The Structure of Lie Groups, Holden-Day, San Francisco, 1965.

10. J. R. Liukkonen and M. W. Mislove, Symmetry in Fourier-Stieltjes algebras, Math. Annalen, 217 (1975), 97-112.

11. J. R. McMullen, Extensions of positive-definite functions, Memoirs Amer. Math. Soc., 117 (1972).

12. G. D. Mostow, On an assertion of Weil, Ann. of Math., 54 (1951), 339-344.

13. H. Reiter, Contributions to harmonic analysis III, J. London Math. Soc., 32 (1957), 447-483.

Received July 27, 1976 and in revised form November 4, 1977. This paper was written while the first-named author was a postdoctoral fellow at the University of British Columbia and the second author was a graduate student at the same university.

Istituto di MATEMATICA,

UNIVERSita di Genova

Via L. B. Alberti 4, 16132 Genova, Italy

AND

UNIVERSity of British Columbia

VANCouver, B. C. V6T 1W5, Canada 


\section{PACIFIC JOURNAL OF MATHEMATICS}

\section{EDITORS}

RICHARD ARENS (Managing Editor)

University of California

Los Angeles, California 90024

C. W. CURTIS

University of Oregon

Eugene, OR 97403

C. C. MOORE

University of California

Berkeley, CA 94720
J. DugundjI

Department of Mathematics University of Southern Californı Los Angeles, California 90007

R. Finn and J. Milgram Stanford University Stanford, California 94305

ASSOCIATE EDITORS

E. F. BECKENBACH

B. H. NeUmanN

F. WOLF

K. YoSHIDA

\section{SUPPORTING INSTITUTIONS}

UNIVERSITY OF BRITISH COLUMBIA CALIFORNIA INSTITUTE OF TECHNOLOGY

UNIVERSITY OF CALIFORNIA

MONTANA STATE UNIVERSITY

UNIVERSITY OF NEVADA, RENO

NEW MEXICO STATE UNIVERSITY

OREGON STATE UNIVERSITY

UNIVERSITY OF OREGON
UNIVERSITY OF SOUTHERN CALIFORNIA

STANFORD UNIVERSITY

UNIVERSITY OF HAWAII

UNIVERSITY OF TOKYO

UNIVERSITY OF UTAH

WASHINGTON STATE UNIVERSITY

UNIVERSITY OF WASHINGTON 


\section{Pacific Journal of Mathematics}

\section{Vol. 80, No. $1 \quad$ September, 1979}

Jeroen Bruijning and Jun-iti Nagata, A characterization of covering dimension by

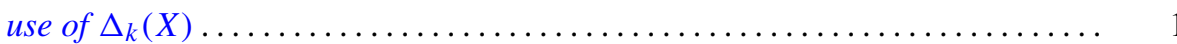

John J. Buoni and Albert Jonathan Klein, On the generalized Calkin algebra ...... 9

Thomas Ashland Chapman, Homotopy conditions which detect simple homotopy

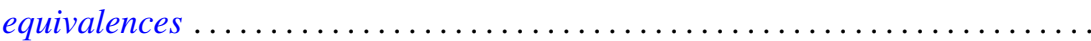

John Albert Chatfield, Solution for an integral equation with continuous interval

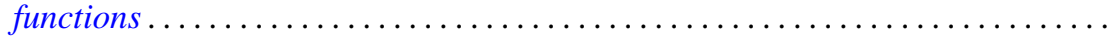

Ajit Kaur Chilana and Ajay Kumar, Spectral synthesis in Segal algebras on

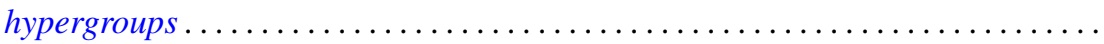

Lung O. Chung, Jiang Luh and Anthony N. Richoux, Derivations and

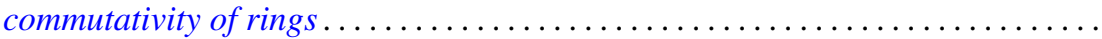

Michael George Cowling and Paul Rodway, Restrictions of certain function spaces to closed subgroups of locally compact groups .....................

David Dixon, The fundamental divisor of normal double points of surfaces........

Hans Georg Feichtinger, Colin C. Graham and Eric Howard Lakien,

Nonfactorization in commutative, weakly selfadjoint Banach algebras . . . . . . .

Michael Freedman, Cancelling 1-handles and some topological imbeddings ....... .

Frank E., III Gerth, The Iwasawa invariant $\mu$ for quadratic fields . . . . . . . . . . . . . .

Maurice Gilmore, Three-dimensional open books constructed from the identity

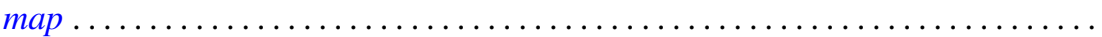

Stanley P. Gudder, A Radon-Nikodým theorem for $*$-algebras .

Peter Wamer Harley, III and George Frank McNulty, When is a point Borel? .

Charles Henry Heiberg, Fourier series with bounded convolution powers . .

Rebecca A. Herb, Characters of averaged discrete series on semisimple real Lie

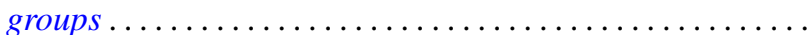

Hideo Imai, On singular indices of rotation free densities . .

Sushil Jajodia, On 2-dimensional CW-complexes with a single 2-cell . . .

Herbert Meyer Kamowitz, Compact operators of the form $u C_{\varphi}$

Matthew Liu and Billy E. Rhoades, Some properties of the Chebyshev method...

213

George Edgar Parker, Semigroups of continuous transformations and generating

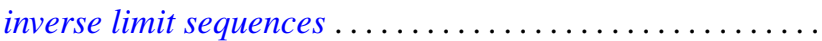

Samuel Murray Rankin, III, Oscillation results for a nonhomogeneous

equation ...

Martin Scharlemann, Transverse Whitehead triangulations ...

Gary Joseph Sherman, A lower bound for the number of conjugacy classes in a

finite nilpotent group

Richard Arthur Shoop, The Lebesgue constants for $\left(f, d_{n}\right)$-summability .

Stuart Jay Sidney, Functions which operate on the real part of a uniform

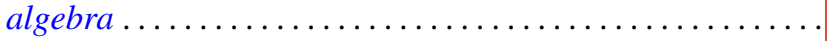

Tim Eden Traynor, The group-valued Lebesgue decomposition 